\title{
Tumor necrosis factor receptor 2 promotes growth of colorectal cancer via the PI3K/AKT signaling pathway
}

\author{
TAO ZHAO ${ }^{1 *}$, HUIHUI $\mathrm{LI}^{2^{*}}$ and ZIFENG LIU ${ }^{3}$ \\ ${ }^{1}$ Oncology Department, Second People's Hospital of Dongying City, Dongying, Shandong 257335; \\ ${ }^{2}$ Department of Medical Oncology, Shandong Cancer Hospital and Institute, Jinan, Shandong 250017; \\ ${ }^{3}$ Second Department of Liver Failure, 302 Hospital of PLA, Beijing 100000, P.R. China
}

Received July 3, 2015; Accepted August 2, 2016

DOI: $10.3892 / 01.2016 .5403$

\begin{abstract}
Tumor necrosis factor receptor 2 (TNFR2) is the receptor for tumor necrosis factor $\alpha$ (TNF- $\alpha)$. TNFR2 differs from tumor necrosis factor 1 (TNFR1) in various ways and is mainly expressed in hematopoietic and endothelial cells. However, studies about its functions in tumors are limited. The contributions of TNFR2 in colorectal cancer (CRC) remain unknown. In the present study, it was found that TNFR2 was positively associated with Ki67 expression in CRC tissues using immunohistochemistry (IHC), and western blot analysis found that Ki67 was upregulated by overexpressing TNFR2 in SW1116 cells and inhibited by silencing TNFR2 in HT29 cells. Methyl thiazolyl tetrazolium assay found that growth of SW1116 cells overexpressing TNFR2 was significantly increased compared with the control group and that the growth of HT29 cells subsequent to silencing TNFR2 was significantly decreased compared with the control group. Clone formation assay found that more clones were formed in SW1116 cells overexpressing TNFR2 than the control group, and less clones formed in HT29 cells subsequent to silencing TNFR2 than the control group. In addition, western blot analysis found that phosphorylation of protein kinase B (AKT) was activated subsequent to overexpressing TNFR2 in SW1116 cells, and inhibited following silencing of TNFR2 in HT29 cells. Additionally, treatment using LY294002 significantly abrogated the promotion of Ki67 expression, growth and clone formation abilities induced by TNFR2 overexpression in SW1116 cells. All the results suggest that TNFR2 can significantly promote $\mathrm{CRC}$ growth via the phosphoinositide 3-kinase/AKT signaling pathway; this provides evidential support for taking TNFR2 as a new target for CRC treatment.
\end{abstract}

Correspondence to: Mr. Tao Zhao, Oncology Department, Second People's Hospital of Dongying City, 28 Chang Chun Road, Dongying, Shandong 257335, P.R. China

E-mail: weifangatjinan1984@163.com

${ }^{*}$ Contributed equally

Key words: tumor necrosis factor receptor 2, colorectal cancer, proliferation

\section{Introduction}

Colorectal cancer (CRC) has been considered the third most common malignant tumor in the world, with an increasing incidence rate (1). As the development of resection treatment, chemotherapy and radiotherapy in previous years has improved, the prognosis of patients diagnosed at an early stage has been enhanced to a certain degree. However, the prognosis of those with advanced tumors remains poor $(2,3)$. Molecular biology research has increasingly become a popular avenue for cancer therapies. Therefore, to additionally understand the molecular mechanisms of cancer occurrence and progression and to find effective molecular treatment targets is important for basic and clinical researchers $(4,5)$.

Tumor necrosis factor receptor (TNFR) is composed of two different subtypes, TNFR1 and TNFR2, which exhibit 30\% homology at amino-acid level in extracellular, cysteine-rich, and ligand-binding regions and are encoded by different genes (6). TNFR1 is ubiquitously expressed in various cells and mediates apoptosis induced by tumor necrosis factor $\alpha$ (TNF- $\alpha$ ) (7). However, TNFR2 is mainly expressed in hematopoietic and endothelial cells and has been found to be required for anti-inflammation, immunoregulation (8), protection against LPS-induced lung damage (9) and bone fracture healing (10). However, studies about TNFR2 in tumors are limited and the functional properties of TNFR2 in CRC remain unknown.

In the present study, immunohistochemistry (IHC) found that TNFR2 was positively associated with Ki67 expression in CRC tissues; western blot analysis found that TNFR2 promoted Ki67 expression in CRC cells via the phosphoinositide 3-kinase (PI3K)/protein kinase B (AKT) signaling pathway; methyl thiazolyl tetrazolium (MTT) assay and clone formation assay also showed that TNFR2 successfully promoted CRC cell growth and clone formation abilities via the PI3K/AKT signaling pathway. All the results confirmed the pro-growth function of TNFR2 in CRC and provided a new target for CRC treatment.

\section{Materials and methods}

Cell lines and culture. Colorectal cancer cell lines SW1116 and HT29 were purchased from American Type Culture Collection (Manassas, VA, USA). SW1116 and HT29 cells were cultured in RPMI 1640 medium (Invitrogen; Thermo Fisher 
Scientific, Inc., Waltham, MA, USA) and McCoy's 5A Medium (Invitrogen; Thermo Fisher Scientific, Inc.), respectively. The two cell lines were supplemented with $10 \%$ fetal bovine serum (Invitrogen; Thermo Fisher Scientific, Inc.).

Tissue samples. Approved by the review board and ethics committee of the Second People's Hospital of Dongying (Dongying, China), the present study selected 90 primary CRC specimens from CRC patients who had undergone surgery between January 2009 and December 2015 in The Second People's Hospital of Dongying (Dongying, China). No patients received chemotherapy, radiotherapy or immunomodulatory therapy prior to surgery.

$I H C$. Tissue samples were cut into $4 \mu \mathrm{m}$ thickness and deparaffinized in xylene and rehydrated in graded ethanol, then boiled in $10 \mathrm{mmol} / 1$ citrate buffer $(\mathrm{pH} 6.0)$ for $3 \mathrm{~min}$ at $100^{\circ} \mathrm{C}$ for antigen unmasking. The sections were then immersed in $3 \% \mathrm{H}_{2} \mathrm{O}_{2}$ for 10 min to block the endogenous peroxidase and in goat serum blocking solution (ZLI-9022; ZsBio, Beijing, China) for 15 min to block non-specific antigens at room temperature. Subsequent to being incubated at room temperature for $2 \mathrm{~h}$ in rabbit anti-human polyclonal primary antibodies against TNFR2 (catalog no. 19272-1-AP; dilution, 1:400; Proteintech Group Inc., Chicago, IL, USA) and Ki67 (working solution; catalog no. RB-9043; Maixin Biotech Co., Ltd., Fuzhou, China), the sections were washed with phosphate-buffered saline (PBS) and incubated in goat anti-rabbit/mouse IgG H\&L (HRP) polymer (working solution) (catalog no. KIT-9921; Maixin Biotech Co., Ltd.) at room temperature for $30 \mathrm{~min}$. Finally, slides were stained with 3,3'-diaminobenzidine and counterstained with hematoxylin (catalog no. DAB-0031; Maixin Biotech Co., Ltd.). Two independent pathologists, who were blind to clinical parameters and clinical outcomes of patients, performed the staining analysis. The percentage of stained cells was recorded at $\times 400$ magnification in at least 5 random fields. The proportion score represented the estimated fraction of positive staining tumor cells $(0, \leq 25 \% ; 1,26-50 \% ; 2,51-75 \% ; 3,>75 \%)$. The intensity score represented the estimated average staining intensity of positive tumor cells ( 0 , negative; 1 , weak; 2 , moderate; 3 , strong). The expression level of TNFR2 was evaluated using the product of proportion score and intensity score at five fields and mean value was obtained ( $\leq 4$ as low expression, $>4$ as high expression); the expression level of Ki67 was directly evaluated using the proportion score.

Transfection. pEZ-M61/green fluorescent protein (GFP)/TNFR2 or vector control (Shanghai GenePharma Co., Ltd., Shanghai, China) was transfected into SW1116 cells using HP X-treme GENE HP Reagents (Roche Diagnostics, Basel, Switzerland) to upregulate TNFR2 expression; p-GPU6/GFP/Neo/TNFR2, including sequence 1 (TNFR2-sh1) and sequence 2 (TNFR2-sh2), or small hairpin RNA control (Shanghai GenePharma Co.,Ltd.) was transfected into HT29 cells to silence TNFR2 expression. All procedures were assessed according to the manufacturer's protocol. Cells were collected for additional study $48 \mathrm{~h}$ later.

Western blot analysis. Cells were washed twice in PBS and lysed in radioimmunoprecipitation assay buffer containing
$1 \%$ protease inhibitor (Beijing ComWin Biotech Co., Ltd., Beijing, China). Protein concentration was determined by spectrophotometer ND-1000 (NanoDrop Technologies; Thermo Fisher Scientific, Inc.). Equal total protein was separated by sodium dodecyl sulfate polyacrylamide gel electrophoresis and transferred to $0.45 \mu \mathrm{m}$ polyvinylidene fluoride membrane. Subsequent to being blocked in Tris-buffered saline and Tween 20 containing 5\% non-fat dry milk for $1 \mathrm{~h}$ at room temperature, the membrane was incubated in primary antibodies at $4^{\circ} \mathrm{C}$ overnight and in goat anti-rabbit polyclonal horseradish peroxidase (HRP)-conjugated secondary antibody (catalog no. SA00001-2; dilution, 1:5,000; Proteintech Group, Inc., Chicago, IL, USA) at room temperature for $1 \mathrm{~h}$. The proteins on the membrane were then detected by chemiluminescence solution (ratio of HRP substrate luminol reagent and HRP substrate peroxide solution was 1:1; EMD Millipore, Billerica, MA, USA); the band intensity was determined by ImageJ software (National Institutes of Health, Bethesda, MA, USA). The primary rabbit anti-human antibodies were as follows: TNFR2 (polyclonal; catalog no. 19272-1-AP; dilution, 1:1,000; Proteintech Group, Inc.); Ki67 (polyclonal; catalog no. ab15580; dilution, 1:1,000; Abcam, Cambridge, MA, USA); phospho-extracellular signal-related kinases (pERK) 1/2 (polyclonal; catalog no. 2219-1; dilution, 1:1,000; Epitomics, Burlingame, CA, USA); ERK1/2 (monoclonal; catalog no. 4695; dilution, 1:1,000; Cell Signaling Technology, Inc., Danvers, MA, USA); phospho-AKT (monoclonal; catalog no. 9611; dilution, 1:1,000; Cell Signaling Technology, Inc.); AKT (monoclonal; catalog no. 4685; dilution, 1:1,000; Cell Signaling Technology, Inc.); glyceraldehyde 3-phosphate dehydrogenase (polyclonal; catalog no. 10494-1-AP; dilution, 1:5,000; Proteintech Group, Inc.).

MTT assay. Cells were counted and plated in 96-well plates in triplicate at $3 \times 10^{3}$ cells in $100 \mu 1$ medium per well. Cell viability was determined at 24, 48, 72, 96 and $120 \mathrm{~h}$ using MTT assay, according to the manufacturers protocol (Beijing Solarbio Science \& Technology Co., Ltd., Beijing, China). Optical density (OD) values were read at $490 \mathrm{~nm}$ using enzyme-labeled instrument (Bio-Rad Model 680; Bio-Rad Laboratories, Beijing, China) and growth curves were drawn. The OD values at $120 \mathrm{~h}$ were compared. The experiment was repeated three times.

Clone formation assay. Cells were counted and plated in 6 -well plates at a density of $1 \times 10^{3}$ cells in $2 \mathrm{ml}$ medium per well. Medium was refreshed every 3 days. Subsequent to 12 days, cells were fixed using methanol for $30 \mathrm{~min}$ and stained using Giemsa, according to the manufacturer's protocol (Beijing Solarbio Science \& Technology Co., Ltd.), then clones were counted by eye by two independent researchers and images were captured. The experiment was repeated 3 times.

Statistical analysis. SPSS 13.0 software (SPSS Inc., Chicago, IL, USA) was used for statistical analysis. All data were expressed as the mean \pm standard deviation. The differences between the two groups were analyzed using Student's two-tailed t-test. $\mathrm{P}<0.05$ was considered to indicate a statistically significant difference. 


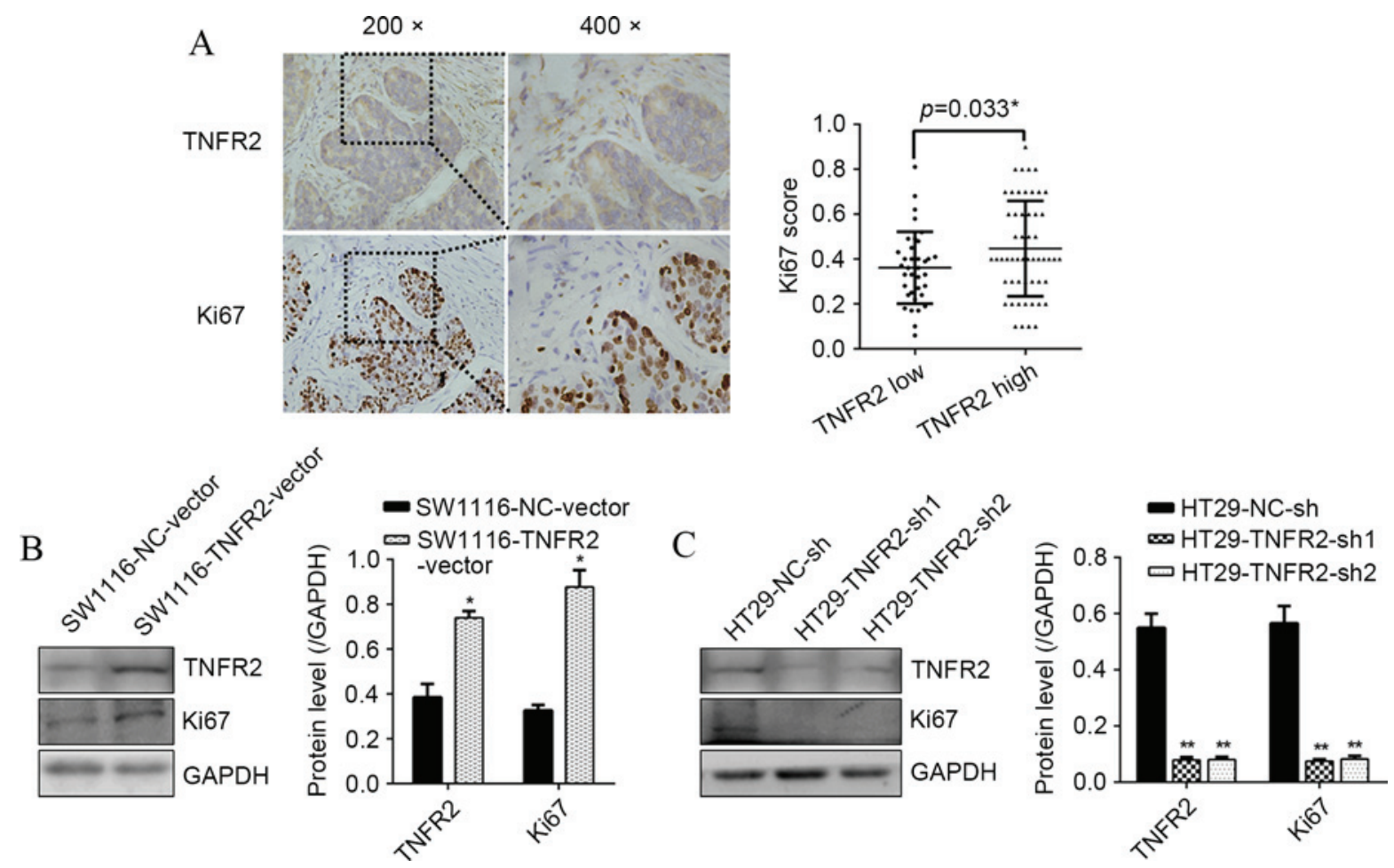

Figure 1. TNFR2 promoted Ki67 expression in CRC tissues and CRC cells. (A) TNFR2 and Ki67 expression in CRC tissues by IHC and statistical analysis. (B) TNFR2 and Ki67 expression in SW1116-NC-vector and SW1116-TNFR2-vector cells, and the quantitative histogram. (C) TNFR2 and Ki67 expression in HT29-NC-sh, HT29-TNFR2-sh1 and HT29-TNFR2-sh2 cells and the quantitative histogram. ${ }^{*} \mathrm{P}<0.05,{ }^{* *} \mathrm{P}<0.01$. TNFR2, tumor necrosis factor receptor 2; CRC, colorectal cancer; IHC, immunohistochemistry; GAPDH, glyceraldehyde 3-phosphate dehydrogenase.

\section{Results}

TNFR2 promoted Ki67 expression in CRC. To confirm the association of TNFR2 with proliferation marker Ki67 in CRC tissues, IHC was assessed. It was found that diffused strong staining of TNFR2 was localized in CRC cell cytoplasm, sporadic staining was also found in the mesenchyme. A positive association between TNFR2 and Ki67 was confirmed $(\mathrm{P}=0.033$; Fig. 1A). At the cellular level, western blot analysis found that subsequent to overexpressing TNFR2 in SW1116 cells, Ki67 was significantly upregulated ( $\mathrm{P}=0.021$; Fig. 1B). Additionally, subsequent to silencing TNFR2 in HT29 cells, Ki67 was significantly downregulated $(\mathrm{P}=0.009$ and $\mathrm{P}=0.008$; Fig. 1C). These results suggest that TNFR2 can promote Ki67 expression in $\mathrm{CRC}$, indicating pro-growth potential.

TNFR2 promoted growth and proliferation of CRC cells. To additionally identify whether TNFR 2 could regulate CRC growth, an MTT assay was assessed. It was found that SW1116 cells overexpressing TNFR2 (SW1116-TNFR2-vector) grew much faster than the control group, with a greater OD value at $120 \mathrm{~h}$ in the MTT assay ( $\mathrm{P}=0.016$; Fig. 2A). The clone forming assay also showed that the clone number of SW1116-TNFR2-vector was 63.00 \pm 15.10 , almost twice that of the control group $(30.33 \pm 9.61)(\mathrm{P}=0.031$; Fig. $2 \mathrm{~B})$. Additionally, HT29 cells with silenced TNFR2 (HT29-TNFR2-sh1 and HT29-TNFR2-sh2) grew much slower than the control group, with a weaker OD value at end time $(120 \mathrm{~h} ; \mathrm{P}=0.036$ and $\mathrm{P}=0.021$; Fig. 2C). The clone forming assay also showed that the clone number of HT29-TNFR2-sh1 and HT29-TNFR2-sh2 was $21.00 \pm 4.58$ and $20 \pm 6.18$, respectively, much less than the control group (41 $\pm 7.21 ; \mathrm{P}=0.027$ and $\mathrm{P}=0.028$; Fig. 2D). All the data suggest that TNFR2 can successfully promote growth and proliferation of CRC cells.

PI3K/AKT was required for growth and proliferation of CRC cells promoted by TNFR2. To additionally confirm the molecular mechanism responsible for regulation of TNFR2 on CRC growth, activation of candidate signal targets was detected by western blot analysis. Overexpressing TNFR2 in SW1116 cells significantly stimulated the phosphorylation of AKT, without change of total AKT, whereas phosphorylation of ERK and total levels of ERK did not alter. In addition, subsequent to silencing TNFR2 in HT29 cells, only the phosphorylation of AKT was inhibited. This indicated that AKT may contribute to regulation of TNFR2 on CRC growth (Fig. 3A and B). To test this hypothesis, the specific AKT inhibitor LY294002 was used. Subsequent to treatment with LY294002, the upregulation of Ki67 induced by TNFR2 was significantly abrogated $(\mathrm{P}=0.017$; Fig. 3C). Additionally, the growth $(\mathrm{P}=0.012$; Fig. 3D) and clone formation ability $(\mathrm{P}=0.033$; Fig. 3E) of the SW1116-TNFR2-vector was also significantly inhibited. The present data confirmed the hypothesis that AKT could contribute to regulation of CRC growth induced by TNFR2.

\section{Discussion}

TNFR2 is the receptor for TNF- $\alpha$. Unlike TNFR1 it does not contain the death domain (DD), and its functions are less understood (11). It is reported that activation of TNFR2 in skin tumors could promote proliferation of tumor cells, not induce apoptosis (12). Tanimura et al (11) reported that TNF- $\alpha$ could 
A

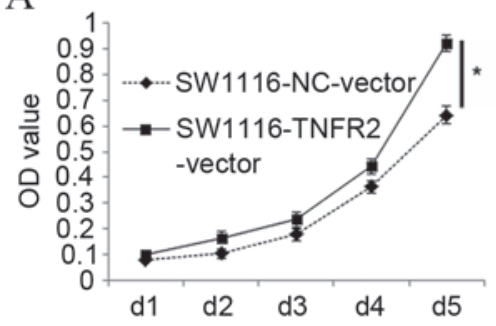

C

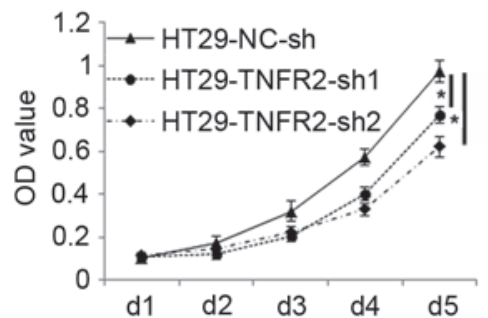

B
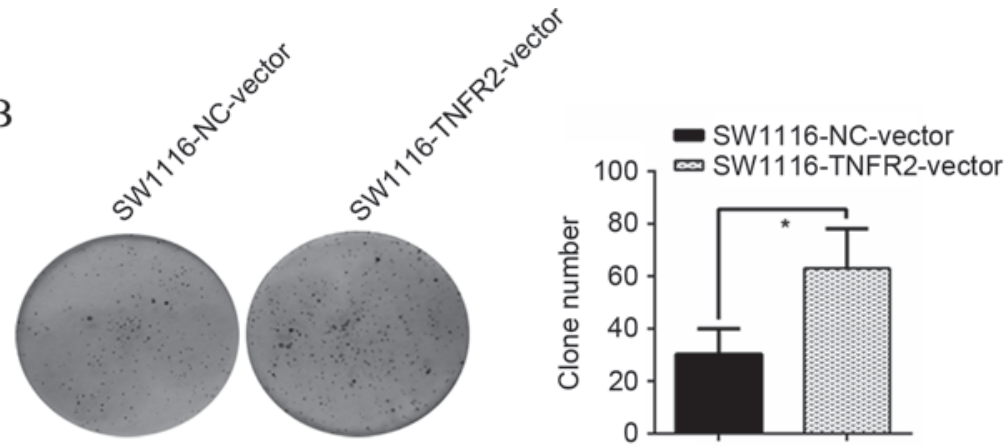

$\mathrm{D}$
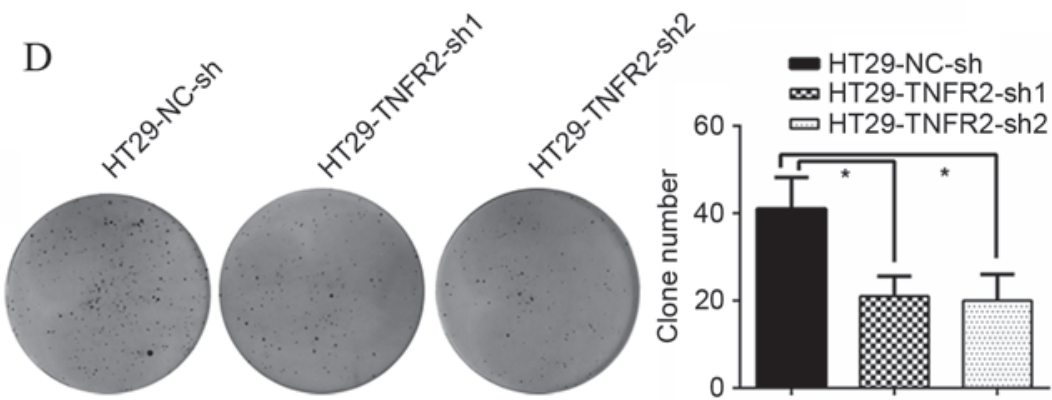

Figure 2. TNFR2 promoted growth and proliferation of CRC cells. (A) Growth curves of SW1116-NC-vector and SW1116-TNFR2-vector cells. (B) Clone formation assay of SW1116-NC-vector and SW1116-TNFR2-vector, and quantitative histogram of clone numbers. (C) Growth curves of HT29-NC-sh, HT29-TNFR2-sh1 and HT29-TNFR2-sh2 cells. (D) Clone formation assay of HT29-NC-sh, HT29-TNFR2-sh1 and HT29-TNFR2-sh2 cells, and quantitative histogram of clone numbers. " $\mathrm{P}<0.05$. TNFR2, tumor necrosis factor receptor 2; CRC, colorectal cancer; OD, optical density.

A

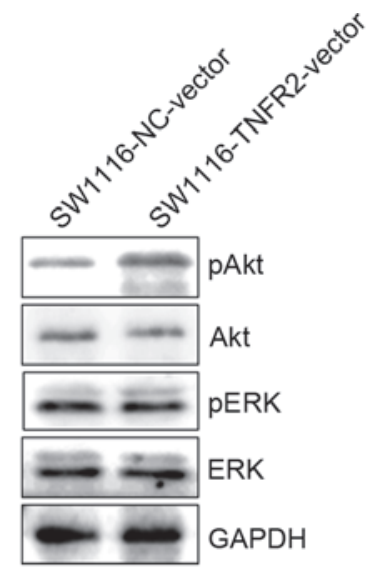

D

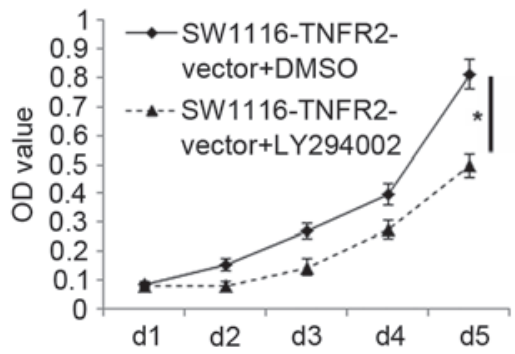

B

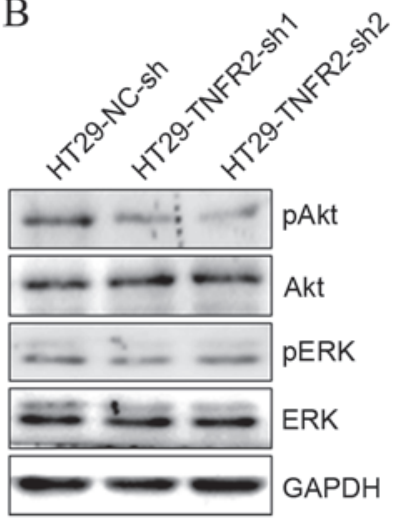

C

\begin{tabular}{rrrrr}
\multicolumn{3}{c}{ SW1116 } \\
\hline- & - & - & + & LY294002 \\
- & - & + & + & DMSO
\end{tabular}

-+++ TNFR2-vector

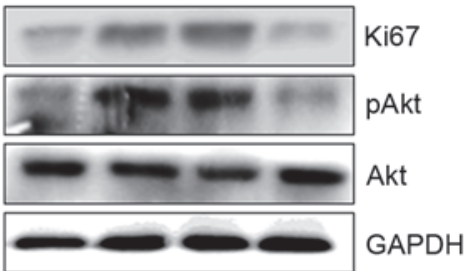

GAPDH

Figure 3. PI3K/AKT was required for the growth and proliferation of CRC cells induced by TNFR2. (A) pAKT, AKT, pERK, ERK expression in SW1116-NC-vector and SW1116-TNFR2-vector cells, as determined by western blot analysis. (B) pAKT, AKT, pERK, ERK expression in HT29-NC-sh, HT29-TNFR2-sh1 and HT29-TNFR2-sh2 cells, as determined by western blot analysis. (C) Ki67 and pAKT upregulation induced by TNFR2 in SW1116 cells was abrogated subsequent to treatment using LY294002. (D) Growth promotion of SW1116 cells induced by TNFR2 was inhibited following treatment using LY294002. (E) Clone formation promotion of SW1116 cells induced by TNFR2 was inhibited subsequent to treatment using LY294002 and quantitative histogram of clone numbers. "P<0.05. PI3K, phosphoinositide 3-kinase; (p)AKT, (phospho)-protein kinase B; (p)ERK, (phospho)-extracellular signal-related kinases; TNFR2, tumor necrosis factor receptor 2; GAPDH, glyceraldehyde 3-phosphate dehydrogenase; DMSO, dimethyl sulfoxide; OD, optical density. 
promote invasiveness of cholangiocarcinoma cells via TNFR2 and Jöhrer et al (13) reported that TNFR2 was required for migration of myeloma cells induced by TNF- $\alpha$. However, the studies by Arnott et al, Baud and Karin, and Grell et al (14-16) reported that, regardless of DD, TNFR2 could also activate death signals and induce cell death by ligand passing to TNFR1. Due to the specific functional properties of TNFR2 in different tumors, the contribution of TNFR2 in CRC remained unknown. In the present study, IHC found that TNFR2 was highly expressed in CRC cells and significantly positively associated with Ki67 expression. Western blot analysis also showed that TNFR2 successfully regulated Ki67 expression, revealing the potential proliferative ability of TNFR2. To additionally verify whether TNFR2 could regulate CRC growth, MTT assay was performed and it was found that upregulating TNFR2 significantly promoted the growth of SW1116 cells. In addition, silencing TNFR2 significantly inhibited the growth of HT29 cells. To test the effect of TNFR2 on cell proliferation ability, clone formation assay was performed and the same trends were identified as found in the MTT assay. This indicates that TNFR2 can promote $\mathrm{CRC}$ growth partly through regulating proliferation ability of CRC cells. This is consistent with the hypothesis that TNFR2 is required for cell proliferation.

Signaling pathways play important roles in the occurrence and progression of tumors and thorough knowledge of signal transduction may provide new targets for tumor therapy. $\mathrm{PI} 3 \mathrm{~K} / \mathrm{AKT}$ and mitogen-activated protein kinase/ERK are important signaling pathways for numerous cellular functions, including proliferation, survival, adhesion and migration (17-22). It has been reported that pretreatment of cells with TNFR2 neutralizing antibody could effectively block the activation of PI3K/AKT and ERK signal pathway in cholangiocarcinoma cells (11). To investigate whether AKT and/or ERK are associated in CRC growth, they were selected as targets for western blot analysis. It was found that TNFR2 upregulation activated phosphorylation of AKT, but not ERK. Furthermore, following treatment using the AKT-specific inhibitor LY294002, not only the upregulation of Ki67 induced by TNFR2 was significantly abrogated, but the growth and clone formation abilities of CRC cells were also inhibited. This was not completely consistent with previous studies in which PI3K/AKT and ERK worked in cholangiocarcinoma. It is possible that the downstream target of TNFR2 in different tumors is type-dependent.

In conclusion, the present study confirmed the contribution of TNFR2 in CRC growth for the first time and additionally elucidated the molecular mechanism. This enriched the understanding of functional properties of TNFR2 and provided evidential support for finding a new promising target for CRC treatment.

\section{References}

1. Becker C, Fantini MC, Wirtz S, Nikolaev A, Lehr HA, Galle PR, Rose-John S and Neurath MF: IL-6 signaling promotes tumor growth in colorectal cancer. Cell Cycle 4: 217-220, 2005.

2. Smith AR, Marquez RT, Tsao WC, Pathak S, Roy A, Ping J, Wilkerson B, Lan L, Meng W, Neufeld KL, et al: Tumor suppressive microRNA-137 negatively regulates Musashi-1 and colorectal cancer progression. Oncotarget 6: 12558-12573, 2015.
3. Siegel R, Ma J, Zou Z and Jemal A: Cancer statistics, 2014. CA Cancer J Clin 64: 9-29, 2014.

4. National Research Council (US) Committee on A Framwework for Developing a New Taxonomy of Disease: Toward Precision Medicine: Building a Knowledge Network for Biomedical Research and a New Taxonomy of Disease. National Academies Press (US), Washington (DC), 2011.

5. Mirnezami R, Nicholson J and Darzi A: Preparing for precision medicine. N Engl J Med 366: 489-491, 2012.

6. Hosono K, Yamada E, Endo H, Takahashi H, Inamori M, Hippo Y, Nakagama $\mathrm{H}$ and Nakajima A: Increased tumor necrosis factor receptor 1 expression in human colorectal adenomas. World J Gastroenterol 18: 5360-5368, 2012.

7. Seitz C, Muller P, Krieg RC, Mannel DN and Hehlgans T: A novel p75TNF receptor isoform mediating NFkappa B activation. J Biol Chem 276: 19390-19395, 2001.

8. Tang W, Lu Y, Tian QY, Zhang Y, Guo FJ, Liu GY, Syed NM, Lai Y, Lin EA, Kong L, et al: The growth factor progranulin binds to TNF receptors and is therapeutic against inflammatory arthritis in mice. Science 332: 478-484, 2011.

9. Guo Z, Li Q, Han Y, Liang Y, Xu Z and Ren T: Prevention of LPS-induced acute lung injury in mice by progranulin. Mediators Inflamm 2012: 540794, 2012

10. Zhao YP, Tian QY, Frenkel S and Liu CJ: The promotion of bone healing by progranulin, a downstream molecule of BMP-2, through interacting with TNF/TNFR signaling. Biomaterials 34 : 6412-6421, 2013.

11. Tanimura Y, Kokuryo T, Tsunoda N, Yamazaki Y, Oda K, Nimura Y, Naing Mon N, Huang P, Nakanuma Y, Chen MF, et al: Tumor necrosis factor alpha promotes invasiveness of cholangiocarcinoma cells via its receptor, TNFR2. Cancer Lett 219: 205-213, 2005.

12. Szlosarek PW and Balkwill FR: Tumour necrosis factor alpha: A potential target for the therapy of solid tumours. Lancet Oncol 4: $565-573,2003$

13. Jöhrer K, Janke K, Krugmann J, Fiegl M and Greil R: Transendothelial migration of myeloma cells is increased by tumor necrosis factor (TNF)-alpha via TNF Receptor 2 and autocrine Up-regulation of MCP-1. Clin Cancer Res 10: 1901-1910, 2004

14. Arnott CH, Scott KA, Moore RJ, Robinson SC, Thompson RG and Balkwill FR: Expression of both TNF-alpha receptor subtypes is essential for optimal skin tumour development. Oncogene 23: 1902-1910, 2004

15. Baud V and Karin M: Signal transduction by tumor necrosis factor and its relatives. Trends Cell Biol 11: 372-377, 2001.

16. Grell M, Zimmermann G, Hülser D, Pfizenmaier K and Scheurich P: TNF receptors TR60 and TR80 can mediate apoptosis via induction of distinct signal pathways. J Immunol 153: 1963-1972, 1994.

17. Dahlmann M, Okhrimenko A, Marcinkowski P, Osterland M, Herrmann P, Smith J, Heizmann CW, Schlag PM and Stein U: RAGE mediates S100A4-induced cell motility via MAPK/ERK and hypoxia signaling and is a prognostic biomarker for human colorectal cancer metastasis. Oncotarget 5: 3220-3233, 2014.

18. Hu SY, Tai CC, Li YH and Wu JL: Progranulin compensates for blocked IGF-1 signaling to promote myotube hypertrophy in $\mathrm{C} 2 \mathrm{C} 12$ myoblasts via the PI3K/Akt/mTOR pathway. FEBS Lett 586: 3485-3492, 2012.

19. Bai T, Lian LH, Wu YL, Wan Y and Nan JX: Thymoquinone attenuates liver fibrosis via PI3K and TLR4 signaling pathways in activated hepatic stellate cells. Int Immunopharmacol 15: 275-281, 2013

20. Li N, Cui J, Duan X, Chen H and Fan F: Suppression of type I collagen expression by miR-29b via PI3K, Akt, and Sp1 pathway in human Tenon's fibroblasts. Invest Ophthalmol Vis Sci 53: 1670-1678, 2012

21. Miao B and Degterev A: Targeting phospshatidylinositol 3-kinase signaling with novel phosphatidylinositol 3,4,5-triphosphate antagonists. Autophagy 7: 650-651,2011.

22. Gentilini A, Marra F, Gentilini $P$ and Pinzani $M$ : Phosphatidylinositol-3 kinase and extracellular signal-regulated kinase mediate the chemotactic and mitogenic effects of insulin-like growth factor-I in human hepatic stellate cells. J Hepatol 32: 227-234, 2000. 